\title{
Clinical risk factors and predictive score for the non-dipper profile in hypertensive patients: a case-control study
}

\author{
Chavalit Chotruangnapa(D), Titima Tansakun®D and Weranuj Roubsanthisuk* (1)
}

\begin{abstract}
Background: Night-time BP, especially non-dipper, is a stronger predictor of adverse cardiovascular outcomes. Ambulatory blood pressure monitoring (ABPM) is a gold standard for the detection of non-dippers but it often is unavailable and expensive. This study aims to determine clinical risk factors that predict non-dipper.

Methods: An exploratory traditional case-control study, exclusive sampling of control was conducted from January 2013 to September 2018 to explore clinical risk factors associated with non-dippers in hypertensive patients. Subgroup analysis was performed in each treated and untreated hypertensive patient. The parsimonious predictive score for non-dippers was constructed.

Results: The study included 208 hypertensive patients receiving $24 \mathrm{~h}$ ABPM. There were 104 dippers and 104 nondippers. Significant clinical risk factors associated with non-dippers were the age of $\geq 65$ years, average office diastolic blood pressure (DBP), and fasting plasma glucose of $\geq 5.6 \mathrm{mmol} / \mathrm{L}$. Results of subgroup analysis showed that dyslipidemia, history of coronary artery disease, use of angiotensin-converting enzyme inhibitors (ACEls) and direct vasodilators, average office DBP, and serum uric acid were associated with non-dippers in treated hypertensive patients, however, there were no risk factors associated with non-dippers in the untreated group. The predictive score for non-dippers in treated group included average office DBP, dyslipidemia, serum uric acid, male, calcium channel blockers and ACEls use. The area under Receiver Operating Characteristic (AuROC) was 0.723. A cut-off point which was $\geq 0.0701$ and prevalence of non-dippers of $46 \%$, this score had a sensitivity of $77.4 \%$, specificity of $65.6 \%$, positive predictive value (PPV) of $66.1 \%$, and negative predictive value (NPV) of $79.6 \%$. For untreated group, age, hemoglobin and body mass index were included in the predictive model. AuROC was 0.74 . There was a sensitivity of $51.9 \%$, specificity of $91.2 \%$, PPV of $82.4 \%$, and NPV of $70.5 \%$ at the cut-off point of $\geq 0.357$, and prevalence of $44 \%$.
\end{abstract}

Conclusion: There were several significant clinical risk factors associated with non-dippers in treated hypertensive patients. The predictive score might be useful for the detection of non-dippers; however, it cannot replace ABPM.

Keywords: Hypertension, Non-dippers, Risk factors, Blood pressure monitoring, Ambulatory

\footnotetext{
*Correspondence: weranuj.rou@gmail.com

Division of Hypertension, Department of Medicine, Faculty of Medicine Siriraj Hospital, Mahidol University, 2 Wanglang Road, Siriraj, Bangkoknoi, Bangkok 10700, Thailand
}

\section{$\triangle B M C$}

(c) The Author(s). 2021 Open Access This article is licensed under a Creative Commons Attribution 4.0 International License, which permits use, sharing, adaptation, distribution and reproduction in any medium or format, as long as you give appropriate credit to the original author(s) and the source, provide a link to the Creative Commons licence, and indicate if changes were made. The images or other third party material in this article are included in the article's Creative Commons. licence, unless indicated otherwise in a credit line to the material. If material is not included in the article's Creative Commons licence and your intended use is not permitted by statutory regulation or exceeds the permitted use, you will need to obtain permission directly from the copyright holder. To view a copy of this licence, visit http://creativecommons.org/licenses/by/4.0/ The Creative Commons Public Domain Dedication waiver (http://creativecommons.org/publicdomain/zero/1.0/) applies to the data made available in this article, unless otherwise stated in a credit line to the data. 


\section{Introduction}

Hypertension is a cause of death globally, accounting for 10.4 million death per year [1]. Uncontrolled hypertension is one of the most important risk factors for cardiovascular disease as well as increases morbidity and mortality $[2,3]$. Blood pressure (BP) normally decreases during sleep by $10-20 \%$ of the average daytime BP in the normal population [4]. The nocturnal BP dipping can be calculated from the equation which was nocturnal BP dipping $(\%)=[$ (average daytime SBP - average nocturnal SBP)/ average daytime SBP] $\times 100$. it is divided into four groups: extreme dippers (nocturnal BP dipping $>20 \%$ ), dippers (nocturnal BP dipping 10-20\%), nondippers (nocturnal BP dipping $0-<10 \%$ ) and reverse dipper (nocturnal BP dipping $<0 \%$ ).

Ambulatory blood pressure monitoring (ABPM) is the best method for the detection of non-dippers and is a better predictor of hypertension-mediated organ damage (HMOD) than office BP $[4,5]$. Previous studies found that night-time BP was a stronger predictor of adverse cardiovascular outcomes than daytime BP [5-8]. Nondippers were related to sleep disturbance, obstructive sleep apnea, obesity, high salt intake, orthostatic hypotension, autonomic dysfunction, chronic kidney disease, diabetic neuropathy, metabolic syndrome, and old age [7-9].

However, ABPM is not widely available and has a high cost in Thailand. There were attempts to find the biomarkers for the prediction of non-dippers to replace ABPM. As the study of Gunebakmaz $\mathrm{O}$ et al. showed that a higher level of red cell distribution width (RDW) was related to non-dippers significantly [10] and Sunbul $\mathrm{M}$ et al. found that non-dippers had significantly higher neutrophil to lymphocyte ratio (NLR) and platelet to lymphocyte ratio (PLR) [11].

This study aimed to explore clinical risk factors and biomarkers that predicted non-dippers and to construct predictive scores for non-dippers.

\section{Materials and methods}

\section{Study population and outcomes}

This present study was a case-control study was conducted from a retrospective chart review in a hypertension clinic in Siriraj hospital from January 2013 to September 2018. The inclusion criteria were as follows: age was at least 18 years, underwent a $24 \mathrm{~h}$ ambulatory BP monitoring and have been diagnosed hypertension on one of the following criteria: 1) office systolic blood pressure $(\mathrm{SBP}) \geq 140 \mathrm{mmHg}$ and/or diastolic blood pressure $(\mathrm{DBP}) \geq 90 \mathrm{mmHg}$ or 2 ) home $\mathrm{SBP} \geq 135 \mathrm{mmHg}$ and/or $\mathrm{DBP} \geq 85 \mathrm{mmHg}$ or 3) ABPM: daytime mean $\mathrm{SBP} \geq 135 \mathrm{mmHg}$ and/or $\mathrm{DBP} \geq 85 \mathrm{mmHg}$ or nighttime mean $\mathrm{SBP}>120 \mathrm{mmHg}$ and $/$ or $\mathrm{DBP}>70 \mathrm{mmHg}$ or $24 \mathrm{~h}$ mean $\mathrm{SBP} \geq 130 \mathrm{mmHg}$ and/or $\mathrm{DBP} \geq 80 \mathrm{mmHg}$ or received anti-hypertensive medications. The exclusion criteria were as follows: pregnancy, peritoneal dialysis or hemodialysis, patients who received an ABPM for $24 \mathrm{~h}$ while admission. All patients underwent a 24h ABPM for evaluation of dipping status. The patients who had at least 10\% of nocturnal BP dipping were classified as dippers and the others were classified as non-dippers. In this study duration, there were 357 patients underwent a 24 h ABPM in Siriraj hospital. One hundred seventyeight of these patients were non-dippers (Supplementary Table 1). The 104 cases were the patients who had nondipping profiles and were randomly selected by computer. Patients with dippers were defined as controls. All controls were randomly selected by the exclusive sampling method at the end of the study from the same source of the cases. Hence, we included 208 patients (matching the 1:1 ratio of case and control) in this study. Because we mainly aimed to investigate the differences of RDW, NLR, and PLR, which might be new biomarkers for non-dippers as in previous studies [10, 11], between two groups. The previous study revealed that RDW of non-dippers was $14.1 \pm 1.33 \%$ while RDW in the other group was $13.58 \pm 0.89 \%$. The total sample size estimation for each group was 104 with $90 \%$ power using the 5\%-level two-sided test for detection of a mean difference of independence between two groups.

Data were collected from patient medical records at the last visit before ABPM included demographic data such as age, body mass index (BMI), current smoking, co-morbidities such as dyslipidemia, diabetes mellitus, ischemic stroke, cardiovascular disease, chronic kidney disease, renal artery stenosis, obstructive sleep apnea, thyroid disease, Cushing syndrome, primary aldosteronism, pheochromocytoma, and aortic disease. Diabetes mellitus was defined as fasting plasma glucose levels that were more than $7 \mathrm{mmol} / \mathrm{L}$ in multiple measurements, previously diagnosed diabetes mellitus, or the use of anti-diabetic medications. Dyslipidemia was defined as serum total cholesterol $>5.2 \mathrm{mmol} / \mathrm{L}$, serum triglyceride $>1.7 \mathrm{mmol} / \mathrm{L}$, low-density lipoprotein cholesterol $>3.4$ $\mathrm{mmol} / \mathrm{L}$, previously diagnosed dyslipidemia, or use of lipid-lowering medications. Complete blood counts, which included total white blood cells, neutrophils, lymphocytes, hemoglobin, hematocrit, red cell distribution width (RDW), and platelets were obtained at the nearest time of performing $24 \mathrm{~h} \mathrm{ABMP}$. Neutrophil-lymphocyte ratio (NLR) and platelet-lymphocyte ratio (PLR) were calculated as the ratio of neutrophil count to lymphocyte count and as the ratio of platelet count to lymphocyte count. The renal function included BUN, creatinine, estimated glomerular filtration rate (eGFR), urine protein to creatinine ratio (UPCR), urine albumin to creatinine ratio (UACR). Stage of CKD, microalbuminuria and macroalbuminuria were classified as KDIGO 2012. 
Fasting plasma glucose, HbA1c, lipid profile and uric acid were collected, as well.

The study was approved by the Institutional Review Board of Faculty of Medicine Siriraj Hospital, Mahidol University (Certificate of Approval No. Si 108/2019).

\section{Statistical analysis}

The demographic data of both cases and controls were presented and analyzed to compare clinical characteristics between both study groups. Continuous data were expressed as mean \pm standard deviation or median and interquartile range, while categorical data are presented as counts and percentages. Chi-square test or Fisher exact test was used for comparison of categorical variables, while student t-test or Mann-Whitney $U$ test was used to compare continuous variables depending on the distribution of data. Univariable logistic regression analysis was performed to determine which risk factors were associated with non-dipper status. Then, multivariable logistic regression was used for exploratory modeling to identify the independent risk factors for non-dippers. The selected parameters in this model consisted of the parameters that had the significant association of nondipper from the univariable analysis. For the prevention of collinearity of the multivariable analysis model, we selected more significant collinear parameters. We also analyzed subgroups that were stratified by the treatment status of these patients. A $p$-value of less than 0.05 was considered statistically significant.

We used forward stepwise logistic regression to construct the 2 separate parsimonious models for nondippers prediction of both treated and untreated hypertensive patients. Potential predictors were the variables with a $p$-value of less than 0.2 from the univariable logistic regression model and the important risk factors from the literature review. Receiver operating characteristic (ROC) curve analysis was performed to determine a cutoff score to predict the non-dipping status. The cut-off score was selected by using Youden index analysis. Model performances were presented with sensitivity, specificity, positive predictive value, and negative predictive value. Statistical analyses were performed using SPSS version 18.0 Chicago: SPSS Inc.

\section{Results}

Of the 208 adult hypertensive patients who underwent $24 \mathrm{~h}, \mathrm{ABPM}$ were enrolled in our study. All patients were divided into two groups: 104 dippers (42.3\% of males, mean age was $53.5 \pm 16.9$ years old) and 104 nondippers ( $31.7 \%$ of males, mean age was $63 \pm 15$ years old) The baseline characteristics of all participants in the two groups were shown in Table 1 (Supplementary Table 1 presented demographic data of our cohort). Non-dippers had significantly diabetes mellitus and dyslipidemia more than dippers. Non-dippers tended to use more numbers of anti-hypertensive medications but this difference did not reach statistical significance. The non-dipping group took calcium channel blockers (CCBs) and beta-blockers (BBs) as anti-hypertensive treatment more than another group. According to the definition of non-dipping status, night-time SBP was higher in non-dippers. In addition, patients with non-dippers had significantly lower all average office, mean of $24 \mathrm{~h}$, daytime and night-time DBP as shown in Table 2.

Determination of factors associated with non-dippers by using univariable logistic regression analysis was presented in Table 3. Exploratory modeling was analyzed by using multivariable logistic regression for exploration of independent risk factors of non-dippers as shown in Table 4. The independent risk factors for non-dippers were age of $\geq 65$ years (odds ratio 2.31, 95\% confident interval (CI) 1.10-4.82), average office DBP (odds ratio 0.96, 95\% CI 0.94-0.99) and impaired fasting plasma glucose (fasting plasma glucose was $>5.6 \mathrm{mmol} / \mathrm{L}$ ) (odds ratio $2.15,95 \%$ CI $1.04-4.47$ ).

\section{Subgroup analysis by treatment status of hypertension}

All dipping and non-dipping hypertensive patients were stratified into treated and untreated hypertensive groups. There were 147 patients in the treated hypertensive group. In this group, there were 70 and 77 patients with dipping and non-dipping status respectively. On the other hand, 61 patients were classified in the untreated hypertensive group. It consisted of 34 dippers and 27 non-dippers. The demographic data of both groups were presented in Supplementary Tables 2, 3, 4 and 5. For treated hypertensive group, dyslipidemia was positively associated with non-dippers (odds ratio 11.73, 95\% CI 1.79-77.64) while the history of coronary artery disease (odds ratio 0.03 , 95\% CI $0.00-0.92$ ), use of angiotensinconverting enzyme inhibitor (odds ratio 0.08, 95\% CI $0.01-0.57$ ) and direct vasodilators (odds ratio $0.04,95 \%$ CI 0.00-0.74), average office DBP (odds ratio 0.89, 95\% CI 0.82-0.96), as well as serum uric acid (odds ratio $0.62,95 \%$ CI $0.41-0.93$ ), had a negative association with non-dippers (Univariable analysis was shown in Supplementary Table 6 and multivariable analysis was shown in Table 5). Evening anti-hypertensive medications administration was the higher proportion in patients with coronary artery disease (11\% versus $1.4 \%$ of patients without coronary artery disease; $p$-value $=0.017$ ). Non-dippers who were treated by direct vasodilators were less frequently taken in the evening than dippers (16.7\% of non-dippers versus $83.3 \%$ of dippers; $p$-value $=0.009$ ). Losartan was used as a hypertensive treatment in $17 \%$ of non-dippers (13 patients) and $2 \%$ of dippers ( 1 patient) ( $p$-value $=0.001$ ) However, there were not independent risk factors for nondippers in untreated hypertensive groups (Univariable 
Table 1 Demographic characteristics of all 208 patients

\begin{tabular}{|c|c|c|c|c|}
\hline Characteristics & $\begin{array}{l}\text { Total } \\
(n=208)\end{array}$ & $\begin{array}{l}\text { Dippers } \\
(n=104)\end{array}$ & $\begin{array}{l}\text { Non-dippers } \\
(n=104)\end{array}$ & $P$-value \\
\hline Age (years) & $58.2 \pm 16.7$ & $53.5 \pm 16.9$ & $63.0 \pm 15.0$ & $<0.001$ \\
\hline Male n (\%) & $77(37)$ & $44(42.3)$ & $33(31.7)$ & 0.114 \\
\hline Body weight (kg) & $65.6 \pm 13.4$ & $67.3 \pm 14.0$ & $63.9 \pm 12.6$ & 0.066 \\
\hline BMI $\left(\mathrm{kg} / \mathrm{m}^{2}\right)^{*}$ & $24.6(21.7,27.5)$ & $24.7(21.8,27.6)$ & $24.2(21.5,27.2)$ & 0.523 \\
\hline Smoking n (\%) & $10(4.8)$ & $10(9.6)$ & $0(0)$ & 0.001 \\
\hline \multicolumn{5}{|l|}{ Co-morbidities n (\%) } \\
\hline Diabetes mellitus & $47(22.6)$ & $13(12.5)$ & $34(32.7)$ & $<0.001$ \\
\hline Dyslipidemia & $122(58.7)$ & $50(48.1)$ & $72(69.2)$ & 0.002 \\
\hline Obstructive sleep apnea & $15(7.2)$ & $8(7.7)$ & $7(6.7)$ & 0.789 \\
\hline Ischemic stroke & $12(5.8)$ & $2(1.9)$ & $10(9.6)$ & 0.017 \\
\hline Coronary artery disease & $10(4.8)$ & $4(3.8)$ & $6(5.8)$ & 0.517 \\
\hline Heart failure & $1(0.5)$ & $1(1)$ & $0(0)$ & 1.000 \\
\hline Chronic kidney disease & $38(18.3)$ & $13(12.5)$ & $25(24)$ & 0.031 \\
\hline $\begin{array}{l}\text { Number and type of anti-hypertensive } \\
\text { medications } n(\%)\end{array}$ & & & & 0.497 \\
\hline 1 & $50(24)$ & $24(23.1)$ & $26(25)$ & \\
\hline 2 & $39(18.8)$ & $22(21.2)$ & $17(16.3)$ & \\
\hline 3 & $33(15.9)$ & $15(14.4)$ & $18(17.3)$ & \\
\hline 4 & $19(9.1)$ & $6(5.8)$ & $13(12.5)$ & \\
\hline 5 & $6(2.9)$ & $3(2.9)$ & $3(2.9)$ & \\
\hline Diuretics & $28(13.5)$ & $15(14.4)$ & $13(12.5)$ & 0.685 \\
\hline CCBs & $95(45.7)$ & $40(38.5)$ & $55(52.9)$ & 0.037 \\
\hline ACEls & $34(16.3)$ & $18(17.3)$ & $16(15.4)$ & 0.708 \\
\hline ARBs & $66(31.7)$ & $30(28.8)$ & $36(34.6)$ & 0.371 \\
\hline Beta blockers & $57(27.4)$ & $22(21.2)$ & $35(33.7)$ & 0.043 \\
\hline Peripheral alpha-1 blockers & $27(13)$ & $11(10.6)$ & $16(15.4)$ & 0.302 \\
\hline Central acting alpha-2 agonists & $9(4.3)$ & $4(3.8)$ & $5(4.8)$ & 1.000 \\
\hline Direct vasodilators & $13(6.2)$ & $10(9.6)$ & $3(2.9)$ & 0.045 \\
\hline \multicolumn{5}{|l|}{ Laboratory results } \\
\hline Hemoglobin $(g / L)^{a}$ & $132(120,145)$ & $134(125,148)$ & $130(117,140)$ & 0.006 \\
\hline Hematocrit (\%) & $40.6 \pm 4.8$ & $41.6 \pm 4.5$ & $39.7 \pm 4.8$ & 0.005 \\
\hline RDW $(\%)^{a}$ & $13.6(12.8,14.6)$ & $13.4(12.7,14.6)$ & $13.7(12.9,14.6)$ & 0.225 \\
\hline $\operatorname{MCV}(\mathrm{fl})^{\mathrm{a}}$ & $88.5(82.2,91.9)$ & $87.8(80.9,91.0)$ & $89.1(84.0,92.0)$ & 0.162 \\
\hline$N L R^{a}$ & $1.80(1.33,2.44)$ & $1.79(1.29,2.50)$ & $1.85(1.42,2.36)$ & 0.623 \\
\hline$P L R^{a}$ & $123.25(97.18,161.71)$ & $128.39(98.91,166.90)$ & $120.95(95.09,158.13)$ & 0.353 \\
\hline Fasting plasma glucose $(\mathrm{mmol} / \mathrm{L})^{a}$ & $5.5(5.1,6.3)$ & $5.4(4.9,5.9)$ & $5.8(5.3,6.4)$ & $<0.001$ \\
\hline $\mathrm{HbA1c}(\%)^{\mathrm{a}}$ & $6.0(5.6,6.5)$ & $6.0(5.7,6.4)$ & $6.0(5.6,6.6)$ & 0.655 \\
\hline Cholesterol (mmol/L) & $4.72 \pm 0.91$ & $4.84 \pm 0.89$ & $4.61 \pm 0.92$ & 0.060 \\
\hline Triglyceride $(\mathrm{mmol} / \mathrm{L})^{\mathrm{a}}$ & $1.12(0.88,1.58)$ & $1.15(0.92,1.71)$ & $1.11(0.87,1.55)$ & 0.495 \\
\hline HDL-cholesterol (mmol/L) & $1.53 \pm 0.44$ & $1.51 \pm 0.47$ & $1.55 \pm 0.41$ & 0.560 \\
\hline LDL-cholesterol (mmol/L) & $2.61 \pm 0.84$ & $2.72 \pm 0.86$ & $2.50 \pm 0.80$ & 0.054 \\
\hline Uric acid (mmol/L) & $0.35 \pm 0.10$ & $0.36 \pm 0.11$ & $0.33 \pm 0.10$ & 0.128 \\
\hline Abnormal proteinuria n (\%) & $55(26.4)$ & $20(19.2)$ & $35(33.7)$ & 0.346 \\
\hline
\end{tabular}


Table 1 Demographic characteristics of all 208 patients (Continued)

\begin{tabular}{llllc}
\hline Characteristics & $\begin{array}{l}\text { Total } \\
(\boldsymbol{n}=\mathbf{2 0 8})\end{array}$ & $\begin{array}{l}\text { Dippers } \\
(\boldsymbol{n}=\mathbf{1 0 4})\end{array}$ & $\begin{array}{l}\text { Non-dippers } \\
(\boldsymbol{n}=\mathbf{1 0 4})\end{array}$ \\
\hline Serum creatinine $(\mu \mathrm{mol} / \mathrm{L})^{\mathrm{a}}$ & $76.02(64.53,97.24)$ & $76.02(63.65,94.59)$ & $76.02(64.53,103.43)$ \\
eGFR $\left(\mathrm{ml} / \mathrm{min} / 1.73 \mathrm{~m}^{2}\right)$ & $80.22 \pm 24.25$ & $84.69 \pm 22.98$ & $75.75 \pm 24.76$ \\
\hline
\end{tabular}

$B M I$ body mass index, $C C B$ s calcium channel blockers, $A C E l s$ angiotensin converting enzyme inhibitors, $A R B s$ angiotensin II receptor blockers, $R D W$ red blood cell distribution width, MCV mean cell volume, NLR neutrophil-lymphocyte ratio, PLR platelet-lymphocyte ratio, HbA1c hemoglobin A1c, HDL high density lipoprotein $L D L$ low density lipoprotein, eGFR estimated glomerular filtration rate

apresented as median and 25 th, 75 th percentile

analysis was shown in Supplementary Table 7, and multivariable analysis was shown in Table 6).

\section{The predictive score for non-dipper stratified by hypertensive treatment status}

The forward step-wise logistic regression analysis with potential predictive factors was performed to construct two predictive models. For treated hypertensive group, the predictive model for non-dippers was $7-(0.081 \mathrm{x}$ average office DBP $)+(1.474 \mathrm{x}$ dyslipidemia $)-(0.297 \mathrm{x}$ serum uric acid $)+(1.031 \mathrm{x}$ use of calcium channel blockers $)$ - $(0.986 \mathrm{x}$ use of angiotensin-converting enzyme inhibitors $)+(0.746 \mathrm{x}$ male gender $)$. Betacoefficient of these prognostic factors and scoring for calculation this formula was presented in Table 7 and Supplementary Figure 1, respectively. The area under Receiver Operating Characteristic (AuROC) was 0.723 (Fig. 1). A cut-off point which was $\geq 0.0701$ and prevalence of non-dippers of $46 \%$, this score had a sensitivity of $77.4 \%$, specificity of $65.6 \%$, positive predictive value (PPV) of $66.1 \%$, and negative predictive value (NPV) of $79.6 \%$.

The predictive model for non-dippers in untreated hypertensive group was $5.443+(0.030 \mathrm{x}$ age $)-(0.379 \mathrm{x}$ hemoglobin $)$ - (0.0933 x body mass index). Table 8 and Supplementary Figure 2 showed beta-coefficient of this prognostic factors and scoring for calculation this formula, respectively. AuROC was 0.74 (Fig. 2). There was sensitivity of $51.9 \%$, specificity of $91.2 \%$, PPV of $82.4 \%$ and NPV of $70.5 \%$ at cut-off point of $\geq 0.357$ and prevalence of $44 \%$.

\section{Discussion}

Our study's finding revealed that the clinical risk factors associated with non-dippers and the mathematical model for the predictive score of hypertensive patients with non-dipping status. The independently associated risk factors for non-dipping status in our study were the elderly (age of $\geq 65$ years) and impaired fasting plasma glucose (fasting plasma glucose was $>5.6 \mathrm{mmol} / \mathrm{L}$ ). In contrast, average office DBP was negatively associated with non-dippers because of the high prevalence of isolated systolic hypertension. These findings were in accordance with the results of the study of Alejandro de la Sierra et al. that analyzed factors associated with blunted night-time BP dipping by using data from the Spanish Society of Hypertension Ambulatory Blood Pressure Monitoring Registry which obtained 24h ABPM data from 42,947 hypertensive patients. They showed that advanced age, obesity, DM, and overt cardiovascular and renal disease were associated with non-dippers [12]. The elderly has high diurnal BP variability because of arterial stiffness and autonomic failure. After 60-70 years of age, $24 \mathrm{~h}$ SBP predominantly increases while $24 \mathrm{~h}$ DBP slightly decreases so isolated systolic hypertension is prevalent in elderly people [8]. This could be the reason why low average office DBP related to non-dippers. Hyperglycemia, including impaired fasting plasma glucose and DM, was found to be associated with nondippers because hyperinsulinism in an insulin-resistant state causes sodium retention and alteration of arterial structure and function. Furthermore, poor glycemic control will result in autonomic dysregulation [13].

Table 2 Hemodynamic data of study population

\begin{tabular}{|c|c|c|c|c|}
\hline Blood pressure & $\begin{array}{l}\text { Total } \\
(n=208)\end{array}$ & $\begin{array}{l}\text { Dippers } \\
(n=104)\end{array}$ & $\begin{array}{l}\text { Non-dippers } \\
(n=104)\end{array}$ & $P$-value \\
\hline Average office SBP (mmHg) & $147.3 \pm 17.2$ & $147.7 \pm 17.4$ & $146.8 \pm 17.0$ & 0.719 \\
\hline Average office DBP (mmHg) & $83.1 \pm 11.9$ & $86.0 \pm 12.2$ & $80.2 \pm 10.8$ & $<0.001$ \\
\hline $24 \mathrm{~h}$ average SBP $(\mathrm{mmHg})$ & $128.6 \pm 14.7$ & $128.3 \pm 14.1$ & $128.9 \pm 15.4$ & 0.782 \\
\hline 24hourrs average DBP (mmHg) & $71.5 \pm 10.9$ & $73.6 \pm 11.9$ & $69.4 \pm 9.4$ & 0.005 \\
\hline Daytime SBP (mmHg) & $132.2 \pm 15.5$ & $134.6 \pm 14.9$ & $129.7 \pm 15.8$ & 0.022 \\
\hline Daytime DBP (mmHg) & $73.9 \pm 12.1$ & $77.8 \pm 12.8$ & $70.0 \pm 9.9$ & $<0.001$ \\
\hline Nighttime SBP (mmHg) & $121.1 \pm 17.1$ & $114.5 \pm 13.5$ & $127.7 \pm 17.9$ & $<0.001$ \\
\hline Nighttime DBP (mmHg) & $66.4 \pm 11.2$ & $64.5 \pm 11.3$ & $68.2 \pm 10.8$ & 0.017 \\
\hline
\end{tabular}

SBP systolic blood pressure, DBP diastolic blood pressure 
Table 3 Univariable logistic regression analysis for evaluation of the association between risk factors and non-dippers

\begin{tabular}{|c|c|c|}
\hline \multirow[t]{2}{*}{ Factors } & \multicolumn{2}{|c|}{ Univariable analysis } \\
\hline & $\begin{array}{l}\text { Odds ratio } \\
\text { (95\% Confident } \\
\text { Interval) }\end{array}$ & $P$ value \\
\hline Age & $1.04(1.02-1.06)$ & $<0.001$ \\
\hline Age $\geq 65$ years & $3.33(1.86-5.97)$ & $<0.001$ \\
\hline Male & $0.63(0.36-1.12)$ & 0.115 \\
\hline Bodyweight & $0.98(0.96-1.00)$ & 0.069 \\
\hline BMl & $0.98(0.93-1.04)$ & 0.069 \\
\hline Diabetes mellitus & $3.40(1.67-6.92)$ & 0.001 \\
\hline Dyslipidemia & $2.43(1.38-4.28)$ & 0.002 \\
\hline Obstructive sleep apnea & $0.87(0.30-2.48)$ & 0.789 \\
\hline Ischemic stroke & $5.43(1.16-25.41)$ & 0.032 \\
\hline Coronary artery disease & $1.53(0.42-5.59)$ & 0.520 \\
\hline Chronic kidney disease & $2.22(1.06-4.62)$ & 0.034 \\
\hline Diuretics & $0.85(0.38-1.88)$ & 0.848 \\
\hline CCBs & $1.80(1.03-3.12)$ & 0.037 \\
\hline ACEls & $0.87(0.42-1.81)$ & 0.708 \\
\hline ARBs & $1.31(0.73-2.35)$ & 0.372 \\
\hline Beta-blockers & $1.89(1.02-3.52)$ & 0.045 \\
\hline Peripheral alpha-1 blockers & $1.54(0.68-3.49)$ & 0.305 \\
\hline Central acting alpha-2 agonists & $1.26(0.33-4.84)$ & 0.734 \\
\hline Direct vasodilators & $0.28(0.08-1.05)$ & 0.058 \\
\hline Use of $\geq 1$ anti-HT medications & $1.39(0.76-2.52)$ & 0.287 \\
\hline Average office SBP & $1.00(0.98-1.01)$ & 0.718 \\
\hline Average office DBP & $0.96(0.93-0.98)$ & 0.001 \\
\hline Hemoglobin & $0.78(0.66-0.93)$ & 0.004 \\
\hline Hematocrit & $0.92(0.86-0.98)$ & 0.006 \\
\hline RDW & $1.01(0.86-1.19)$ & 0.923 \\
\hline MCV & $1.01(0.98-1.04)$ & 0.392 \\
\hline NLR & $1.10(0.88-1.37)$ & 0.405 \\
\hline PLR & $1.00(1.00-1.01)$ & 0.314 \\
\hline Fasting plasma glucose & $1.02(1.01-1.04)$ & 0.006 \\
\hline Fasting plasma glucose $\geq 5.6 \mathrm{mmol} / \mathrm{L}$ & $2.74(1.56-4.81)$ & $<0.001$ \\
\hline $\mathrm{HbA} 1 \mathrm{c}$ & $1.40(0.93-2.11)$ & 0.112 \\
\hline Cholesterol & $0.99(0.99-1.000)$ & 0.062 \\
\hline Triglyceride & $1.00(0.99-1.00)$ & 0.269 \\
\hline HDL-cholesterol & $1.01(0.98-1.02)$ & 0.558 \\
\hline LDL-cholesterol & $0.99(0.98-1.00)$ & 0.055 \\
\hline Uric acid & $0.87(0.72-1.04)$ & 0.129 \\
\hline Abnormal proteinuria & $1.37(0.71-2.64)$ & 0.346 \\
\hline Serum creatinine & $1.19(0.61-2.32)$ & 0.620 \\
\hline eGFR & $0.98(0.973-1.00)$ & 0.009 \\
\hline
\end{tabular}

$B M I$ body mass index, CCBs calcium channel blockers, ACEls angiotensin converting enzyme inhibitors, $A R B s$ angiotensin II receptor blockers, RDW red blood cell distribution width, MCV mean cell volume, NLR neutrophillymphocyte ratio, PLR platelet-lymphocyte ratio, HbA1c hemoglobin A1c, $H D L$ high density lipoprotein, $L D L$ low density lipoprotein, eGFR estimated glomerular filtration rate
Table 4 Exploratory model by using multivariable logistic regression analysis for evaluation of the association between independent risk factors and non-dippers

\begin{tabular}{|c|c|c|}
\hline \multirow[t]{2}{*}{ Factors } & \multicolumn{2}{|c|}{ Multivariable analysis } \\
\hline & $\begin{array}{l}\text { Odds ratio } \\
\text { (95\% Confident } \\
\text { Interval) }\end{array}$ & $P$ value \\
\hline Age $\geq 65$ years & $2.31(1.10-4.82)$ & 0.026 \\
\hline Diabetes mellitus & $1.10(0.43-2.82)$ & 0.846 \\
\hline Dyslipidemia & $1.45(0.71-2.94)$ & 0.309 \\
\hline Ischemic stroke & $3.85(0.70-21.22)$ & 0.122 \\
\hline CCBs & $1.02(0.49-2.09)$ & 0.966 \\
\hline Beta blockers & $1.07(0.50-2.28)$ & 0.863 \\
\hline Average office DBP & $0.96(0.94-0.99)$ & 0.016 \\
\hline Hemoglobin & $0.85(0.70-1.03)$ & 0.090 \\
\hline $\begin{array}{l}\text { Fasting plasma glucose } \\
\geq 5.6 \mathrm{mmol} / \mathrm{L}\end{array}$ & $2.15(1.04-4.47)$ & 0.040 \\
\hline eGFR & $1.00(0.99-1.02)$ & 0.721 \\
\hline
\end{tabular}

Although some evidence supported that hypertensive patients with non-dippers had increase platelet activation and a high inflammatory state [14], the inflammatory biomarkers, which were RDW, MCV, NLR, and PLR, in our study did not significantly associate with nondipping status. Our results contrasted with Gunebakmaz $\mathrm{O}$ et al. and Sunbul M et al.'s ones because of the different study populations' characteristics. The study of Gunebakmz $\mathrm{O}$ et al. included age and gender matching between the subjects with and without hypertension [10]. The inclusion criteria in the study of Gunebakmz $\mathrm{O}$ et al. and Sunbul $\mathrm{M}$ et al. were similar but only subjects with hypertension were included in the study of Sunbul M et al. [11]. Both previous studies excluded the subjects with heart disease (e.g. coronary artery disease, chronic heart failure), cerebrovascular disease, hematologic disorders (e.g. anemia, thrombocytopenia), malignancy, renal or hepatic dysfunction, secondary hypertension, autoimmune disease and systemic inflammation. Although the inclusion criteria of our study were similar to both previous studies, we did not exclude patients with cardiovascular disease, stroke, kidney disease and hematologic problem (for example anemia). Even though daytime SBP in non-dippers was lower than dippers, nighttime blood pressure phenotype still predicted adverse cardiovascular outcomes and was not dependent on daytime blood pressure. This hypothesis was supported by the study of Hong-Qi Fan, et al. which showed that isolated nocturnal hypertension predicted cardiovascular outcome [15]. We considered the effect of antihypertensive medication on dipping status; hence we analyzed the association of risk factors and non- 
Table 5 Exploratory model by using multivariable logistic regression analysis for evaluation of the association between independent risk factors and non-dippers in the treated hypertensive group

\begin{tabular}{|c|c|c|}
\hline \multirow[t]{2}{*}{ Factors } & \multicolumn{2}{|l|}{ Multivariable analysis } \\
\hline & $\begin{array}{l}\text { Odds ratio } \\
(95 \% \mathrm{Cl})\end{array}$ & $P$ value \\
\hline Age & $1.011(0.952-1.074)$ & 0.721 \\
\hline Male & $4.320(0.611-30.564)$ & 0.143 \\
\hline BMI & $0.978(0.845-1.133)$ & 0.770 \\
\hline Diabetes mellitus & $2.483(0.347-17.764)$ & 0.365 \\
\hline Dyslipidemia & $11.773(1.785-77.641)$ & 0.010 \\
\hline Obstructive sleep apnea & $1.505(0.069-32.933)$ & 0.795 \\
\hline Coronary artery disease & $0.031(0.001-0.924)$ & 0.045 \\
\hline Diuretics & $0.224(0.026-1.938)$ & 0.174 \\
\hline CCBs & $1.788(0.365-8.757)$ & 0.473 \\
\hline ACEls & $0.076(0.010-0.572)$ & 0.012 \\
\hline ARBs & $0.423(0.081-2.197)$ & 0.306 \\
\hline Beta-blockers & $2.091(0.486-9.003)$ & 0.322 \\
\hline Peripheral alpha-1 blockers & $1.446(0.175-11.932)$ & 0.732 \\
\hline Central acting alpha-2 agonists & $0.737(0.028-19.498)$ & 0.855 \\
\hline Direct vasodilators & $0.038(0.002-0.743)$ & 0.031 \\
\hline Evening drug administration & $1.584(0.395-6.355)$ & 0.517 \\
\hline Average office SBP & $1.052(0.992-1.115)$ & 0.092 \\
\hline Average office DBP & $0.886(0.818-0.959)$ & 0.003 \\
\hline Hemoglobin & $0.843(0.507-1.399)$ & 0.508 \\
\hline RDW & $0.670(0.401-1.120)$ & 0.126 \\
\hline MCV & $0.941(0.842-1.052)$ & 0.287 \\
\hline NLR & $0.949(0.386-2.331)$ & 0.909 \\
\hline PLR & $1.001(0.995-1.007)$ & 0.760 \\
\hline Fasting plasma glucose & $0.972(0.934-1.012)$ & 0.168 \\
\hline Cholesterol & $0.987(0.910-1.069)$ & 0.744 \\
\hline Triglyceride & $0.996(0.971-1.024)$ & 0.818 \\
\hline HDL-cholesterol & $0.980(0.893-1.075)$ & 0.672 \\
\hline LDL-cholesterol & $1.014(0.939-1.095)$ & 0.720 \\
\hline Uric acid & $0.615(0.407-0.932)$ & 0.022 \\
\hline Abnormal proteinuria & $1.552(0.306-7.871)$ & 0.596 \\
\hline eGFR & $0.999(0.953-1.047)$ & 0.966 \\
\hline
\end{tabular}

$B M I$ body mass index, CCBs calcium channel blockers, ACEls angiotensin converting enzyme inhibitors, $A R B$ s angiotensin II receptor blockers, $R D W$ red blood cell distribution width, MCV mean cell volume, NLR neutrophillymphocyte ratio, $P L R$ platelet-lymphocyte ratio, $H b A 1 c$ hemoglobin $A 1 c, H D L$ high density lipoprotein, $L D L$ low density lipoprotein, eGFR estimated glomerular filtration rate

dippers stratified by treatment status of hypertensive patients. For the treated hypertensive group, dyslipidemia was associated with non-dippers because dyslipidemia is an atherosclerotic risk factor of cardiovascular disease and is associated with metabolic syndrome. A study by Sipping Dai, et al. showed that non-dipping hypertensive
Table 6 Exploratory model by using multivariable logistic regression analysis for evaluation of the association between independent risk factors and non-dippers in the untreated hypertensive group

\begin{tabular}{|c|c|c|}
\hline \multirow[t]{2}{*}{ Factors } & \multicolumn{2}{|l|}{ Multivariable analysis } \\
\hline & $\begin{array}{l}\text { Odds ratio } \\
(95 \% \mathrm{Cl})\end{array}$ & $P$ value \\
\hline Age & $1.030(0.917-1.157)$ & 0.616 \\
\hline Male & $1.200(0.069-20.840)$ & 0.901 \\
\hline BMl & $1.055(0.857-1.298)$ & 0.614 \\
\hline Diabetes mellitus & $0.022(0.000-6.512)$ & 0.189 \\
\hline Dyslipidemia & 0.669 (0.019-23.167) & 0.824 \\
\hline Average office SBP & $0.943(0.866-1.027)$ & 0.175 \\
\hline Average office DBP & 1.087 (0.954-1.239) & 0.209 \\
\hline Hemoglobin & $0.285(0.073-1.117)$ & 0.072 \\
\hline RDW & $1.046(0.358-3.056)$ & 0.935 \\
\hline MCV & $1.257(0.944-1.675)$ & 0.117 \\
\hline NLR & $0.660(0.153-2.846)$ & 0.577 \\
\hline PLR & $1.032(0.991-1.074)$ & 0.129 \\
\hline Fasting plasma glucose & $1.036(0.943-1.140)$ & 0.459 \\
\hline Cholesterol & $1.051(0.688-1.606)$ & 0.817 \\
\hline Triglyceride & 0.999 (0.914-1.092) & 0.985 \\
\hline HDL-cholesterol & $0.894(0.595-1.341)$ & 0.588 \\
\hline LDL-cholesterol & $0.932(0.614-1.417)$ & 0.744 \\
\hline eGFR & $1.006(0.896-1.130)$ & 0.916 \\
\hline Abnormal proteinuria & $2.216(0.033-151.082)$ & 0.712 \\
\hline
\end{tabular}

patients with dyslipidemia were associated with cardiovascular disease [16]. Nevertheless, history of coronary artery disease, use of angiotensin-converting enzyme inhibitor and direct vasodilators as well as serum uric acid had a negative relation with non-dippers. Because of the

Table $7 \beta$ co-efficient of the parameters of the predictive model for non-dippers by using forward stepwise logistic regression in the treated hypertensive group

\begin{tabular}{lll}
\hline Factors & $\boldsymbol{\beta}$ co-efficient & $\boldsymbol{P}$-value \\
\hline Average office DBP & -0.081 & 0.001 \\
Dyslipidemia & 1.474 & 0.008 \\
Serum uric acid & -0.297 & 0.046 \\
Use of CCBs & 1.031 & 0.059 \\
Use of ACEls & -0.986 & 0.087 \\
Male & 0.746 & 0.172 \\
Constant & 7.000 & 0.001 \\
\hline
\end{tabular}

CCBs calcium channel blockers, $A C E l s$ angiotensin-converting enzyme inhibitors

* P-value of less than 0.2 was considered statistically significant for this predictive model 


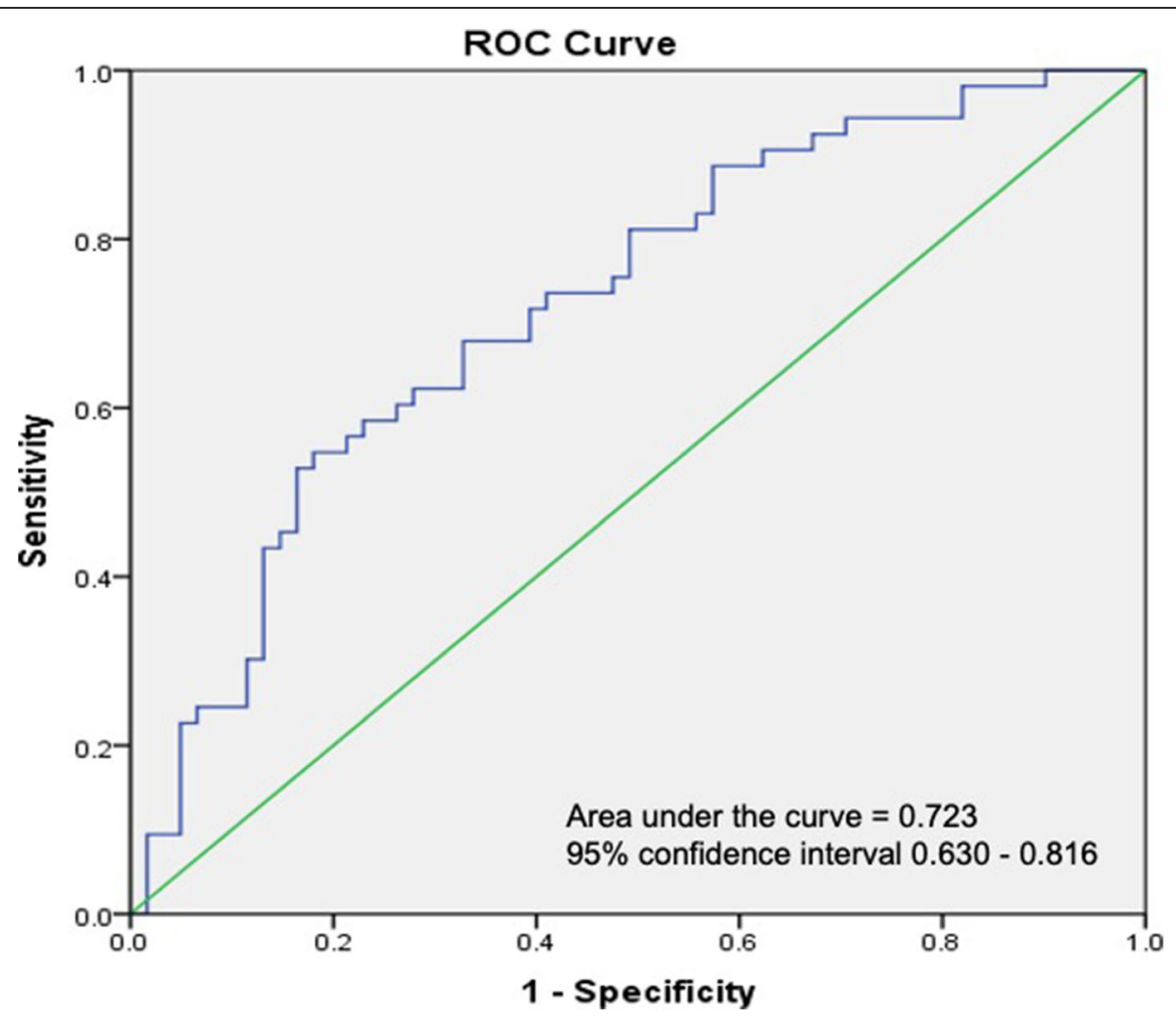

Fig. 1 Receiver Operating Characteristic (ROC) analysis of predictive score model for non-dippers in treated hypertensive patients

high proportion of evening administration of antihypertensive medications in patients with coronary artery disease and the ones who took direct vasodilators, we supposed that evening administration of direct vasodilators was able to decrease night-time BP. It was supported by previous studies such as the Hygia Chronotherapy trial of Hermida RC et al. [17] demonstrated the efficacy of blood pressure lowering drugs at bedtime on improvement of blood pressure control and dipping status. But the exact mechanism of using direct vasodilators themselves negatively related to non-dippers was unknown. The evidence of the efficacy of direct vasodilators on dipping status and nocturnal blood pressure control is also limited. We suggested that further research to determine which blood pressure lowering

Table $8 \beta$ co-efficient of the parameters of the predictive model for non-dippers by using forward stepwise logistic regression in the untreated hypertensive group

\begin{tabular}{lll}
\hline Factors & $\boldsymbol{\beta}$ co-efficient & $\boldsymbol{P}$-value* \\
\hline Age & 0.030 & 0.114 \\
Body mass index & -0.093 & 0.146 \\
Hemoglobin & -0.379 & 0.064 \\
Constant & 5.443 & 0.120
\end{tabular}

* $P$-value of less than 0.2 was considered statistically significant for this predictive model drugs should be taken in the evening for the treatment of non-dipping status and the mechanistic explanation of those drugs are required. Some studies revealed that serum uric acid was associated with non-dipping status $[18,19]$. Giallauria $\mathrm{F}$ et al. and Turak $\mathrm{O}$ et al.'s ones demonstrated the positive association of serum uric acid and non-dipping circadian pattern in newly diagnosed untreated hypertensive patients without treatments affected uric acid metabolism (such as allopurinol) in order to control the confounders $[18,19]$. Serum uric acid in our study was negatively associated with nondippers because it might be modified by the uricosuric effect of losartan, which was taken in non-dippers more than dippers. Uric acid lowering property of losartan had been shown in several studies [20-22]. So losartan was the important confounder that might cause the negative association between serum uric acid and nondipping status in our study.

It is well known that blood pressure from ABPM is a better predictor for cardiovascular and renal disease than office BP. ABPM can provide nocturnal blood pressure, which is correlated with adverse cardiovascular and renal outcomes. Several hypertension guidelines including Thai hypertension guideline suggest considering ABPM as one method of out of office BP measurement. In Thailand, $24 \mathrm{~h} \mathrm{ABPM}$ is currently the only method to 


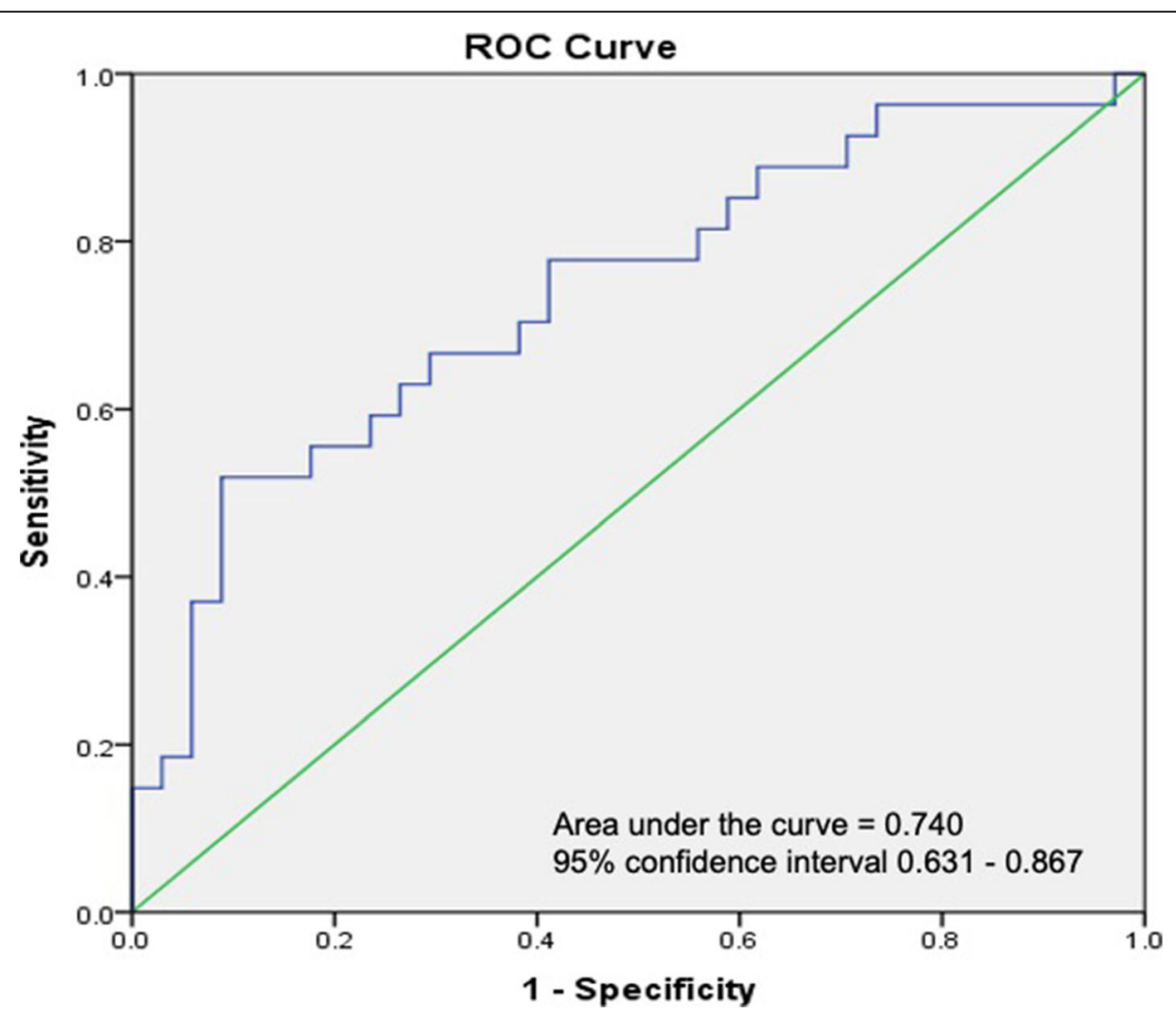

Fig. 2 Receiver Operating Characteristic (ROC) analysis of predictive score model for non-dippers in untreated hypertensive patients

evaluate night-time BP but it is not widely available due to the high cost of ABPM devices. Current evidence supports that non-dippers are related to hypertensionmediated organ damage [23-26]. In addition, extreme dippers also result in myocardial and brain ischemia at night time [27, 28]. Therefore, precise diagnosis of nondippers in hypertensive patients will specifically lead to improvement of blood pressure control and reduction of adverse cardiovascular events. We proposed two new mathematical models for the prediction of non-dipping status by using clinical data and basic investigation in the treated and untreated hypertensive group. By using forward stepwise multivariable logistic regression for constriction of predictive score, the usages of calcium channel blockers (CCBs) and angiotensin-converting enzyme inhibitors (ACEIs) reached the statistical significance that we had decided. In addition, ACEIs were effective in decreasing nocturnal blood pressure, especially when given at bedtime [29]. The pathophysiology of non-dipping is complex. It may involve sodium retention, activation of sympathetic nervous system and renin-angiotensin-aldosterone system [30-32]. Dihydropyridine calcium channel blockers are the mainstay of the treatment of hypertension. The studies of dihydropyridine calcium channel blockers treatment and non-dipping hypertensive patients had mixed results depending on pharmacokinetics, timing of administration and patients' profiles (e.g. dipping status, resistant hypertension, etc.) [33-35]. Luo Y et al.'s study demonstrated that $24 \mathrm{~h} \mathrm{BP}$ profiles were normalized by taking amlodipine at the evening time [35]. In contrast with this study, all treated hypertensive patients in our study took CCBs in the morning. We did not know the exact mechanism why ACEIs or CCBs attenuate non-dipping status and further investigations to clarify these associations are required.

The performance of our predictive models had fair accuracy. There are no standard cut-off points so we used the Youden index statistic for the selection of cut-off points. Nevertheless, the overall accuracy of the predictive model was not as good as ABPM. It might result from a small sample size. Hence, our predictive model cannot replace ABPM. Further study with a larger sample size is needed to improve the accuracy of the predictive model and perform external validity.

\section{Strengths and limitations}

This study showed the risk factors which were independent predictors of non-dipping status in hypertensive patients and provided the first parsimonious predictive model for Thai hypertensive patients. These models can be applied in both hypertensive patients with and without treatment by anti-hypertensive medications because 
separate analysis and modeling were performed according to the treatment status.

There were some limitations in this study. First, missing data and miss-classification bias was the problem in the retrospective study. The interval from data collection of risk factors to ABPM application varied in each participant because of different follow-up intervals and frequency of individual laboratory testing. Thus, it may affect the association between risk factors and outcome. Second, our predictive models need external validation. We have planned to use these predictive models in realworld practice and to re-analyze their accuracy as well as to improve their precision in the next step. Third, there is not enough sample size to perform external validation in this study.

\section{Conclusion}

There were several significant clinical risk factors associated with non-dippers in treated hypertensive patients. The two predictive models stratified by treatment status of hypertension might be useful for the detection of non-dippers in real-world clinical practice, particularly in primary and secondary care hospitals. However, it cannot replace ABPM.

\section{Supplementary Information}

The online version contains supplementary material available at https://doi. org/10.1186/s40885-021-00180-4

Additional file 1: Supplementary Table 1. Baseline characteristics of the cohort. Supplementary Table 2. Demographic characteristics of treated hypertensive patients. Supplementary Table 3. Hemodynamic data of treated hypertensive groups. Supplementary Table 4.

Demographic characteristics of untreated hypertensive patients.

Supplementary Table 5. Hemodynamic data of untreated hypertensive groups. Supplementary Table $\mathbf{6}$. Univariable logistic regression analysis for evaluation of the association between risk factors and non-dippers in the treated hypertensive group. Supplementary Table 7. Univariable logistic regression analysis for evaluation of the association between risk factors and non-dippers in the untreated hypertensive group.

Additional file 2: Supplementary Figure 1. Predictive score model for non-dippers in treated hypertensive patients.

Additional file 3: Supplementary Figure 2. Predictive score model for non-dippers in untreated hypertensive patients.

\section{Acknowledgments}

The authors would like to greatly acknowledge Ms. Khemajira Karaketklang for her skillful and professional data processing and analysis. She works at Research and Academic Service Unit, Department of Medicine, Faculty of Medicine Siriraj Hospital, Mahidol University.

\section{Authors' contributions}

CC conceived the main research idea, participated in the study's design, collected data, performed statistical analysis, and was the main contributor in writing the manuscript. TT contributed to data collection, statistical analysis. WR participated in the study's design and data analysis planning. All authors read and approved the final manuscript.

\section{Funding}

There was no funding for this study.
Availability of data and materials

Not applicable.

\section{Declarations}

\section{Ethics approval and consent to participate}

This study was approved by the Institutional Review Board of Faculty of Medicine Siriraj Hospital, Mahidol University (Certificate of Approval No. Si 108/2019), and data from medical records were permitted by the participants and Siriraj hospital director.

Consent for publication

Not applicable.

\section{Competing interests}

All authors declare that they do not have competing interests.

Received: 3 May 2021 Accepted: 9 September 2021

Published online: 15 November 2021

\section{References}

1. Stanaway G, Afshin A, Gakidou E, et al. Global, regional, and national comparative risk assessment of 84 behavioural, environmental and occupational, and metabolic risks or clusters of risks for 195 countries and territories, 1990-2017: a systematic analysis for the global burden of disease study 2017. Lancet. 2018;392(10159):1923-94.

2. Lewington S, Clarke R, Qizilbash N, Peto R, Collins R. Age-specific relevance of usual blood pressure to vascular mortality: a meta-analysis of individual data for one million adults in 61 prospective studies. Lancet. 2002;360:1903-13.

3. Rapsomaniki E, Timmis A, George J, et al. Blood pressure and incidence of twelve cardiovascular diseases: lifetime risks, healthy life-years lost, and agespecific associations in 1.25 million people. Lancet. 2014;383:1899-911.

4. O'Brien E, Parati G, Stergiou G, et al. European Society of Hypertension position paper on ambulatory blood pressure monitoring. J Hypertens. 2013;31:1731-68.

5. Hermida RC, Smolensky MH, Ayala DE, Portaluppi F. Ambulatory blood pressure monitoring (ABPM) as the reference standard for diagnosis of hypertension and assessment of vascular risk in adults. Chronobiol Int. 2015; 32:1329-42.

6. Mancia G, Fagard R, Narkiewicz K, et al. 2013 ESH/ESC guidelines for the management of arterial hypertension: the task force for the management of arterial hypertension of the European Society of Hypertension (ESH) and of the European Society of Cardiology (ESC). J Hypertens. 2013;31:1281-357.

7. Williams B, Mancia G, Spiering W, et al. 2018 ESC/ESH guidelines for the management of arterial hypertension: the task force for the management of arterial hypertension of the European Society of Cardiology (ESC) and the European Society of Hypertension (ESH). Eur Heart J. 2018;39:3021-104.

8. Parati G, Stergiou G, O'Brien E, et al. European Society of Hypertension practice guidelines for ambulatory blood pressure monitoring. J Hypertens. 2014;32:1359-66.

9. Tilea I, Petra D, Ardeleanu E, Hutanu A, Varga A. Clinical conditions and predictive markers of non-dipper profile in hypertensive patients. Acta Marisiensis Seria Medica. 2018;64:10-6.

10. Gunebakmaz O, Kaya MG, Duran M, Akpek M, Elcik D, Eryol NK. Red blood cell distribution width in 'non-dippers' versus 'dippers'. Cardiology. 2012;123: 154-9.

11. Sunbul M, Gerin F, Durmus E, et al. Neutrophil to lymphocyte and platelet to lymphocyte ratio in patients with dipper versus non-dipper hypertension. Clin Exp Hypertens. 2014;36:217-21.

12. de la Sierra A, Redon J, Banegas JR, et al. Prevalence and factors associated with circadian blood pressure patterns in hypertensive patients. Hypertension. 2009;53:466-72.

13. Kanbay M, Turgut F, Uyar ME, Akcay A, Covic A. Causes and mechanisms of nondipping hypertension. Clin Exp Hypertens. 2008;30:585-97.

14. Kaya MG, Yarlioglues M, Gunebakmaz O, et al. Platelet activation and inflammatory response in patients with non-dipper hypertension. Atherosclerosis. 2010;209:278-82.

15. Fan $H Q$, Li $Y$, Thijs $L$, et al. Prognostic value of isolated nocturnal hypertension on ambulatory measurement in 8711 individuals from 10 populations. J Hypertens. 2010;28:2036-45. 
16. Dai S, Huang B, Zou Y, Liu Y. Associations of dipping and non-dipping hypertension with cardiovascular diseases in patients with dyslipidemia. Arch Med Sci. 2019;15:337-42.

17. Hermida RC, Crespo JJ, Domínguez-Sardiña M, et al. Bedtime hypertension treatment improves cardiovascular risk reduction: the Hygia Chronotherapy trial. Eur Heart J. 2019:41:4565-76.

18. Giallauria F, Predotti P, Casciello A, et al. Serum uric acid is associated with non-dipping circadian pattern in young patients (30-40 years old) with newly diagnosed essential hypertension. Clin Exp Hypertens. 2016;38:233-7.

19. Turak O, Ozcan F, Tok D, et al. Serum uric acid, inflammation, and nondipping circadian pattern in essential hypertension. J Clin Hypertens. 2013;15:7-13.

20. Matsumura $\mathrm{K}$, Arima $\mathrm{H}$, Tominaga $\mathrm{M}$, et al. Effect of losartan on serum uric acid in hypertension treated with a diuretic: the COMFORT study. Clin Exp Hypertens. 2015;37:192-6.

21. Kurniadi A, Mulia DP, Ali Z. Losartan effect decreased uric acid Serum and Urine Levelin Hyperuricemia Hypertensivepatients. J Hypertens. 2015;33:e14.

22. Rayner BL, Trinder YA, Baines D, Isaacs S, Opie LH. Effect of losartan versus candesartan on uric acid, renal function, and fibrinogen in patients with hypertension and Hyperuricemia associated with diuretics*. Am J Hypertens. 2006;19:208-13.

23. Kario K, Pickering TG, Matsuo T, Hoshide S, Schwartz JE, Shimada K. Stroke prognosis and abnormal nocturnal blood pressure falls in older hypertensives. Hypertension. 2001;38:852-7.

24. O'Brien E, Sheridan J, O'Malley K. Dippers and non-dippers. Lancet. 1988;2:397.

25. Shimada K, Kawamoto A, Matsubayashi K, Ozawa T. Silent cerebrovascular disease in the elderly. Correlation with ambulatory pressure. Hypertension. 1990;16:692-9

26. Hoshide S, Kario K, Hoshide Y, et al. Associations between nondipping of nocturnal blood pressure decrease and cardiovascular target organ damage in strictly selected community-dwelling normotensives. Am J Hypertens. 2003:16:434-8

27. Kario K, Pickering TG, Umeda Y, et al. Morning surge in blood pressure as a predictor of silent and clinical cerebrovascular disease in elderly hypertensives: a prospective study. Circulation. 2003;107:1401-6.

28. Pierdomenico SD, Bucci A, Costantini F, Lapenna D, Cuccurullo F, Mezzetti A. Circadian blood pressure changes and myocardial ischemia in hypertensive patients with coronary artery disease. J Am Coll Cardiol. 1998: 31:1627-34

29. Hermida RC, Ayala DE. Chronotherapy with the angiotensin-converting enzyme inhibitor ramipril in essential hypertension: improved blood pressure control with bedtime dosing. Hypertension. 2009;54:40-6.

30. Ragot S, Herpin D, Siché JP, Ingrand P, Mallion JM. Autonomic nervous system activity in dipper and non-dipper essential hypertensive patients. What about sex differences? J Hypertens. 1999;17:1805-11.

31. Osanai T, Okuguchi T, Kamada T, et al. Salt-induced exacerbation of morning surge in blood pressure in patients with essential hypertension. J Hum Hypertens. 2000;14:57-64.

32. Tadic M, Cuspidi C, Grassi G, Mancia G. Isolated nocturnal hypertension: what do we know and what can we do? Integr Blood Press Contr. 2020;13:63-9.

33. Chu CS, Lee KT, Chen SH, et al. Morning versus evening administration of a calcium channel blocker in combination therapy for essential hypertension by ambulatory blood pressure monitoring analysis. Int Heart J. 2005;46:433-42.

34. White WB, Mansoor GA, Pickering TG, et al. Differential effects of morning and evening dosing of nisoldipine ER on circadian blood pressure and heart rate. Am J Hypertens. 1999;12:806-14.

35. Yifan Luo LR, Jiang M, Yang C. Anti-hypertensive efficacy of amlodipine dosing during morning versus evening: a meta-analysis. Rev Cardiovasc Med. 2019;20:91-8.

\section{Publisher's Note}

Springer Nature remains neutral with regard to jurisdictional claims in published maps and institutional affiliations.

\section{Ready to submit your research? Choose BMC and benefit from:}

- fast, convenient online submission

- thorough peer review by experienced researchers in your field

- rapid publication on acceptance

- support for research data, including large and complex data types

- gold Open Access which fosters wider collaboration and increased citations

- maximum visibility for your research: over $100 \mathrm{M}$ website views per year

At BMC, research is always in progress.

Learn more biomedcentral.com/submissions 